\title{
Pancreatite aguda em pacientes etilistas: revisão narrativa
}

\author{
Acute pancreatitis in alcoholic patients: narrative review
}

Pancreatitis aguda en pacientes alcohólicos: revisión narrativa

Joyce de Paula Gomes ${ }^{1 *}$, Isadora Thamires Pacheco Celestino ${ }^{1}$, Amanda Mendes Clemente Vilella ${ }^{2}$, David de Almeida Chaves ${ }^{1}$, Hermes Vinícius Nogueira Neri², Idrys Henrique Leite Guedes ${ }^{3}$, Raquel Melo Vasconcelos ${ }^{2}$, Rodrigo Rodrigues Salgado4, Tayná Alves dos Santos ${ }^{5}$.

\section{RESUMO}

Objetivo: Discorrer acerca da pancreatite aguda de origem alcoólica através de uma revisão da literatura no que tange a sua fisiopatologia, quadro clínico, diagnóstico, abordagens terapêuticas, complicações e prognóstico. Revisão bibliográfica: A pancreatite aguda pode ser definida como um processo inflamatório agudo do pâncreas que pode envolver os tecidos peripancreáticos e/ou órgãos a distância, podendo variar de formas leves a graves. Nesse sentido, destaca-se a de origem alcoólica, responsável por $30 \%$ das pancreatites agudas, afetando predominantemente homens jovens com histórico de consumo alcoólico abusivo. O tratamento é orientado a partir da gravidade do quadro clínico. O prognóstico do paciente está intimamente relacionado a avaliação dos parâmetros clínicos e laboratoriais, bem como os escores Ranson e APACHE-II. Considerações finais: Nota-se uma incidência expressiva de PA alcoólica ( $30 \%$ das PAs) e, desta forma, ressalta-se os impactos de seus efeitos ao indivíduo e ao sistema de saúde, evidenciando a importância de realizar uma atualização dos pontos principais relativos a esse tema.

Palavras-chave: Pancreatite, Alcoolismo, Pancreatite alcoólica.

\section{ABSTRACT}

Objective: Discourse about acute pancreatitis of alcoholic origin through a review of the literature regarding its pathophysiology, clinical picture, diagnosis, therapeutic approaches, complications and prognosis. Literature review: An acute pancreatitis can be defined as an acute inflammatory process of the tissue that can involve peripancreatic tissues and / or distant organs, which can vary from mild forms to graves. In this sense, presenting an alcoholic origin responsible for $30 \%$ of acute pancreatitis, predominantly affecting young men with a history of alcohol abuse. Treatment is guided by the clinical picture. The patient's prognosis is closely related to the evaluation of clinical and laboratory drugs, as well as the Ranson and APACHE-II scores. Final considerations: There is a significant incidence of alcoholic AP (30\% of APs) and, thus, the impacts of its effects on the individual and the health system are highlighted, highlighting the importance of updating the main points related to this theme.

Key words: Pancreatitis, Alcoholism, Alcoholic pancreatitis.

\section{RESUMEN}

Objetivo: Hablar sobre la pancreatitis aguda de origen alcohólico a través de una revisión de la literatura sobre su fisiopatología, cuadro clínico, diagnóstico, abordajes terapéuticos, complicaciones y pronóstico. Revisión bibliográfica: Una pancreatitis aguda se puede definir como un proceso inflamatorio agudo del tejido que puede involucrar tejidos peripancreáticos y / u órganos distantes, que pueden variar de formas leves a tumbas. En este sentido, presenta un origen alcohólico responsable del $30 \%$ de la pancreatitis aguda, que afecta predominantemente a hombres jóvenes con antecedentes de abuso de alcohol. El tratamiento se guía por el

${ }^{1}$ Pontifícia Universidade Católica de Minas Gerais (PUC-MG), Betim - MG. *E-mail: joycedpg@outlook.com

${ }^{2}$ Centro Universitário de Belo Horizonte (UNIBH), Belo Horizonte - MG.

${ }^{3}$ Universidade Federal de Campina Grande (UFCG), Campina Grande - PB.

${ }^{4}$ Universidade do Vale do Sapucaí (UNIVAS), Pouso Alegre - MG.

${ }^{5}$ Universidade Federal de Minas Gerais (UFMG), Belo Horizonte - MG.

SUBMETIDO EM: 6/2020

ACEITO EM: 7/2020

PUBLICADO EM: 10/2020

REAS/EJCH | Vol.12(10) | e4223 | DOI: https://doi.org/10.25248/reas.e4223.2020

Página 1 de 9 
cuadro clínico. El pronóstico del paciente está estrechamente relacionado con la evaluación de fármacos clínicos y de laboratorio, así como con las puntuaciones de Ranson y APACHE-II. Consideraciones finales: Existe una incidencia significativa de PA alcohólica ( $30 \%$ de los PA) y, por lo tanto, se destacan los impactos de sus efectos sobre el individuo y el sistema de salud, destacando la importancia de actualizar los puntos principales relacionados con este tema.

Palabras clave: Pancreatitis, Alcoholismo, Pancreatitis alcohólica.

\section{INTRODUÇÃO}

O pâncreas é uma glândula mista, sua porção exócrina secreta enzimas digestivas e bicarbonato de sódio que são importantes para a digestão de proteínas, carboidratos e gorduras. Já sua porção endócrina secreta insulina, amilina, glucagon, somatostatina e polipeptídeo pancreático (SILVERTORN DU, 2017).

Dentro das patologias pancreáticas podemos citar a pancreatite aguda (PA), definida, de acordo com a classificação de Atlanta, como um processo inflamatório agudo do pâncreas que pode envolver os tecidos peripancreáticos e/ou órgãos a distância, podendo variar de formas leves a graves. A sua fisiopatologia engloba a formação de edema intersticial, infiltração de células inflamatórias e diversos graus de apoptose celular, hemorragias e necrose (ÁLVARES LGGS, et al., 2014).

Essa doença apresenta como principais fatores etiológicos a litíase biliar, o álcool e a hipertrigliceridemia. Menos frequentemente pode ser causada pela hipercalemia e por uso de medicamentos (ZATERKA S e EISIG $J N, 2011$ ). No Brasil, a causa principal é a doença biliar calculosa; álcool e litíase biliar respondem por $80 \%$ dos casos de pancreatite aguda (BRASILEIRO FILHO G, 2016). Com relação à epidemiologia, esta moléstia apresenta incidência de, aproximadamente, 13-45 casos para cada grupo de 100.000 indivíduos ao ano, com existência de variações geográficas (YADAV, 2013).

É importante considerar que o álcool é responsável por $30 \%$ das pancreatites agudas, afetando predominantemente homens jovens com história de consumo alcoólico abusivo (ZATERKA S e EISIG JN, 2011). No Brasil, cerca de $17,9 \%$ da população adulta faz uso abusivo de bebida alcoólica, de acordo com dados da Organização Mundial de Saúde (OMS). Esse consumo expressivo apresenta implicações importantes na saúde pública, tanto a nível nacional quanto mundial. Há uma estimativa, a nível mundial, de cerca de 237 milhões de homens e 46 milhões de mulheres que sofrem com patologias relacionados ao consumo do álcool, com prevalência na população Europeia ( $14,8 \%$ nos homens, 3,5\% nas mulheres) e Americana (11,5\% nos homens e 5,1\% nas mulheres) (OMS, 2018a apud Souza, 2019).

Desse modo, o objetivo do presente estudo é discorrer acerca da pancreatite aguda de origem alcoólica através de uma revisão da literatura abordando sobre a fisiopatologia, o diagnóstico, o manejo clínico terapêutico, as possíveis complicações e o prognóstico.

\section{REVISÃO BIBLIOGRÁFICA}

\section{Fisiopatologia}

A patogênese da PA baseia-se na autodigestão enzimática do parênquima pancreático, causada pelo extravasamento ou ativação excessiva das enzimas. O extravasamento está relacionado à obstrução dos ductos pancreáticos, principalmente por cálculos biliares na papila de Vater, que promovem acúmulo de secreção e consequente aumento da pressão intraductal. Com isso, há comprometimento do fluxo sanguíneo e isquemia capaz de lesar células do parênquima. Com a destruição de células acinares ocorre extravasamento de enzimas, às quais provocam lesões parenquimatosas e iniciam o processo inflamatório. Agressão às células acinares também pode iniciar a pancreatite. Tal agressão é causada sobretudo pelo álcool, mas também por agentes infecciosos, medicamentos, traumatismos e isquemia (AGUILAR A, et al., 2019). 
A autodigestão do parênquima pancreático é o mecanismo mais aceito para explicar as lesões da $P A$, as quais ocorrem por ativação das enzimas digestivas dentro do próprio órgão, em vez de ocorrer apenas na luz intestinal. Em boa parte dos casos, as razões da ativação intrapancreática da tripsina são pouco conhecidas; hipóxia, traumatismos, infecções, endo e exotoxinas podem iniciar a ativação de pró-enzimas pancreáticas. Uma vez ativada, a tripsina, além de promover a autodigestão do parênquima, ativa outras pró-enzimas, como elastase e fosfolipase, que resultam, respectivamente, em lesão vascular (e, em consequência, hemorragia) e esteatonecrose (BRASILEIRO FILHO G, 2016).

A PA ocorre, muitas vezes, após consumo abusivo de álcool, uma vez que o etanol e seus metabólitos têm ação lesiva direta sobre as células acinares, possivelmente pela geração de radicais livres. Essa lesão celular libera citocinas e induz inflamação. Além disso, o álcool estimula a secreção pancreática e promove contração do esfíncter de Oddi, o que aumenta a pressão intraductal. Assim, como o álcool é em parte metabolizado no pâncreas, é aventado que seus metabólitos podem ativar a tripsina dentro das células acinares (SINGH P, GARG $P, 2016)$.

A metabolização do álcool, apesar de conhecidamente ser realizada no fígado, também ocorre no pâncreas, no qual o etanol é submetido a reações oxidativas e não oxidativas. A oxidação por meio das enzimas do complexo citocromo P450, dentre as quais a CYP2E1, é inibida pela reação não oxidativa de metabolização do álcool ingerido, já que a atividade da "Carboxyl ester lipase" (CEL) cresce proporcionalmente à quantidade de álcool presente no organismo e paralelamente a via oxidativa tem sua atividade reduzida. A CEL é responsável pela produção de éster etílico de ácido graxo (FAEE) a partir de etanol e ácido graxo, de forma que o incremento na concentração do FAEE é acompanhado da manutenção de níveis aumentados de cálcio intracelular e de proteínas da matriz extracelular, aspectos relacionados ao dano gerado pelo álcool ao pâncreas.

Outros fatores que provêm da ação do álcool e impactam a integridade pancreática são a ativação de enzimas lisossomais e digestivas, como o tripsinogênio, quando ainda imaturas no interior dos ácinos pancreáticos, causando digestão tecidual, e a ativação do fator de transcrição nuclear kappa B (NF-kB) que gera inflamação no órgão (KLOCHKOV A, et al., 2020).

Apesar desses dados, o conhecimento integral acerca do mecanismo pelo qual o consumo de álcool gera prejuízos ao funcionamento do pâncreas ainda está em construção no meio científico. Persiste a dúvida se a obstrução dos ductos pancreáticos por secreções pancreáticas mais viscosas é causa ou consequência do dano pancreático causado pelo etanol (CHOWDHURY P e GUPTA P, 2006).

\section{Quadro clínico}

O paciente acometido por PA pode ou não apresentar dor abdominal, sendo mais comum a presença de dor na região epigástrica (dor em "faixa") e a irradiação para as costas. O quadro clínico muitas vezes se apresenta através de episódios repentinos de abdome agudo, seguido de progressão da dor para um estado crônico, que se intensifica ao ingerir alimento e é aliviado ao mover-se o tronco póstero-anteriormente, posição conhecida como prece maometana.

Com o crescente acometimento da função pancreática, a insuficiência orgânica em cerca de $90 \%$ dos casos provoca o surgimento de esteatorreia, deficiência de vitaminas lipossolúveis, disfunções exócrinas e, em estágios mais avançados da doença, disfunções endócrinas como diabetes, que ocorre em quase metade dos pacientes (CHATILA AT, et al., 2019).

Alguns achados sistêmicos acompanham os demais sintomas da doença e podem ser úteis ao diagnóstico, como febre, desidratação e taquicardia. Além de achados no exame físico, como o sinal de Blumberg à palpação abdominal nos casos avançados, distensão abdominal decorrente de íleo paralítico e os sinais de Grey-Turner e de Cullen, equimoses em flancos e periumbilical, respectivamente, que surgem entre 24 e 48 horas após a ruptura do parênquima pancreático (BARRY K, 2018; PHAM A e FORSMARK C, 2018; RAHMAN A, et al., 2019). 


\section{Diagnóstico}

O diagnóstico de PA pode ser feito pela dosagem sérica de amilase e lipase. Caso ocorra dúvida no diagnóstico, pode-se realizar exames de imagem, como a tomografia computadorizada de abdome, uma vez que a presença de edema e/ou necrose do parênquima pancreático pode confirmar o caso de pancreatite. Entretanto, os exames de imagem não são compulsórios para concluir o diagnóstico (DOHERTY GM, 2017).

\section{1) Amilase + Lipase séricas:}

A amilase sérica é um exame laboratorial para a detecção de pancreatite aguda. Derivada das células pancreáticas acinares, a amilase sérica aumenta sua concentração nas primeiras 2 a 12 horas após o início de uma pancreatite aguda, atinge os valores de pico em 48 horas e volta ao normal em 3 a 5 dias. Ela possui um valor preditivo positivo para PA de aproximadamente $100 \%$. Já a lipase sérica, também é derivada das células pancreáticas acinares, sua concentração sofre um aumento discreto antes da elevação dos níveis de amilase, 4 a 8 horas após o início de um episódio de pancreatite aguda, atingindo os valores de pico igualmente antes (em 24 horas). Ela também permanece por mais tempo no soro (8 a 14 dias). Por esses motivos, é mais sensível e específica do que a amilase sérica.

Entretanto, existe uma particularidade, diferente de um pâncreas normal que inflama devido a pancreatite biliar, na pancreatite aguda alcoólica, o pâncreas do paciente é alvo de lesões que ocorrem no decorrer de vários anos, com o consumo de bebidas alcoólicas. Isso faz com que a glândula sofre alterações em sua arquitetura, visto que os ductos vão se alterando pela deposição de tampões proteináceos.

Dessa forma, o aumento dos níveis de amilase é afetado, ao contrário da lipase, pois no pâncreas parece haver 4 a 5 vezes mais lipase do que amilase. Portanto, a proporção lipase/amilase é sugerida como exame laboratorial preditivo para pancreatite induzida por álcool. É considerado que uma proporção $>3$ é considerada preditiva, enquanto uma proporção $>5$ é diagnóstica de pancreatite aguda induzida por álcool (MCPHERSON RA e PINCUS MR, 2013).

\section{2) Tomografia Computadorizada Contrastada:}

A TC com contraste venoso possui grande sensibilidade e especificidade. É muito utilizada quando há dúvida diagnóstica (casos de clínica sugestiva, mas a dosagem de amilase e lipase não confirmam o diagnóstico). É o melhor exame de imagem para identificação de complicações locorregionais na pancreatite aguda, por essa razão é indicada em quadros graves. Não é justificado o uso nos casos leves, visto a baixa probabilidade de complicações nessa circunstância.

É necessário evitar a TC contrastada em pacientes cuja pancreatite aguda evolui com insuficiência renal aguda (IRA) para evitar nefropatia induzida por contraste. Nestes casos opta-se preferencialmente pela ressonância eletromagnética (SANTOS JS, et al., 2003).

\section{3) Ressonância magnética (RM):}

A RM é correspondente à TC em termos de acurácia para o diagnóstico de $\mathrm{PA}$, mas a ressonância tem duas vantagens em relação a tomografia: em primeiro lugar, quando há suspeita de pancreatite biliar, a colangioressonância pode identificar mais de $90 \%$ dos cálculos na via biliar. Em segundo lugar, não é preciso expor o paciente ao contraste iodado endovenoso. O ponto fraco desse exame é o alto custo e a baixa disponibilidade. Além disso, a RM é melhor que a TC na investigação de coleções líquidas, com maior capacidade de distinguir pseudocistos, abscesso, necrose, entre outros (SANTOS JS, et al., 2003).

\section{4) Outros exames:}

A ultrassonografia é sempre indicada, pois é o método de escolha para diagnosticar a litíase biliar, causa mais comum de PA. Já a radiografia simples é um exame importante para casos de abdome agudo, em especial quando há dúvida se o abdome é cirúrgico ou não (SANTOS JS, et al., 2003). 


\section{Tratamento}

O manejo da PA é orientado a partir da análise da gravidade do quadro clínico. Desse modo, o estudo laboratorial é essencial para avaliação e condução do paciente. Para isso, realiza-se a interpretação de alguns parâmetros, como: amilase e lipase séricos, cálcio sérico, perfil metabólico, triglicerídeos séricos, hemograma completo e lactato (VEGE SS, 2020). Espera-se achar valores, aproximadamente, três vezes acima do limite superior para amilase e/ou lipase, o que é forte indicativo para a confirmação do diagnóstico de pancreatite. É preferível que se analise os níveis da amilase em relação à lipase no manejo desse indivíduo (CHATILA AT, et al., 2019).

Mesmo com a análise dos parâmetros laboratoriais supracitados, inexiste um teste diagnóstico definitivo ou ideal para a pancreatite. A TC contrastada de abdome e a RM abdominal são os exames de imagem mais fiéis na visualização de acometimento pancreático. Entretanto, sabe-se que tais modalidades não são disponíveis amplamente em todos os serviços de saúde, além de não estarem preconizadas como prática rotineira na abordagem da PA leve. Por outro lado, a realização de ultrassonografia abdominal é recomendada, de preferência nas primeiras 48-72 horas, para todos os pacientes com suspeita de PA, a fim de verificar a presença de cálculos biliares e de complicações pancreáticas, tais como coleção de fluidos e acometimento vascular (CHATILA AT, et al., 2019).

Nesse sentido, é importante discriminar quais pacientes necessitarão de cuidados intensivos e quais podem ser tratados em regime ambulatorial. Essa triagem é fundamental para garantir que os pacientes que realmente necessitam de intervenções especiais consigam ser adequadamente atendidos. Dessa forma, caso o paciente se enquadre no contexto clínico descrito abaixo, ele deve ser admitido na unidade de terapia intensiva (UTI), conforme esquematização da figura abaixo.

Figura 1 - Diagrama para admissão de paciente na UTI

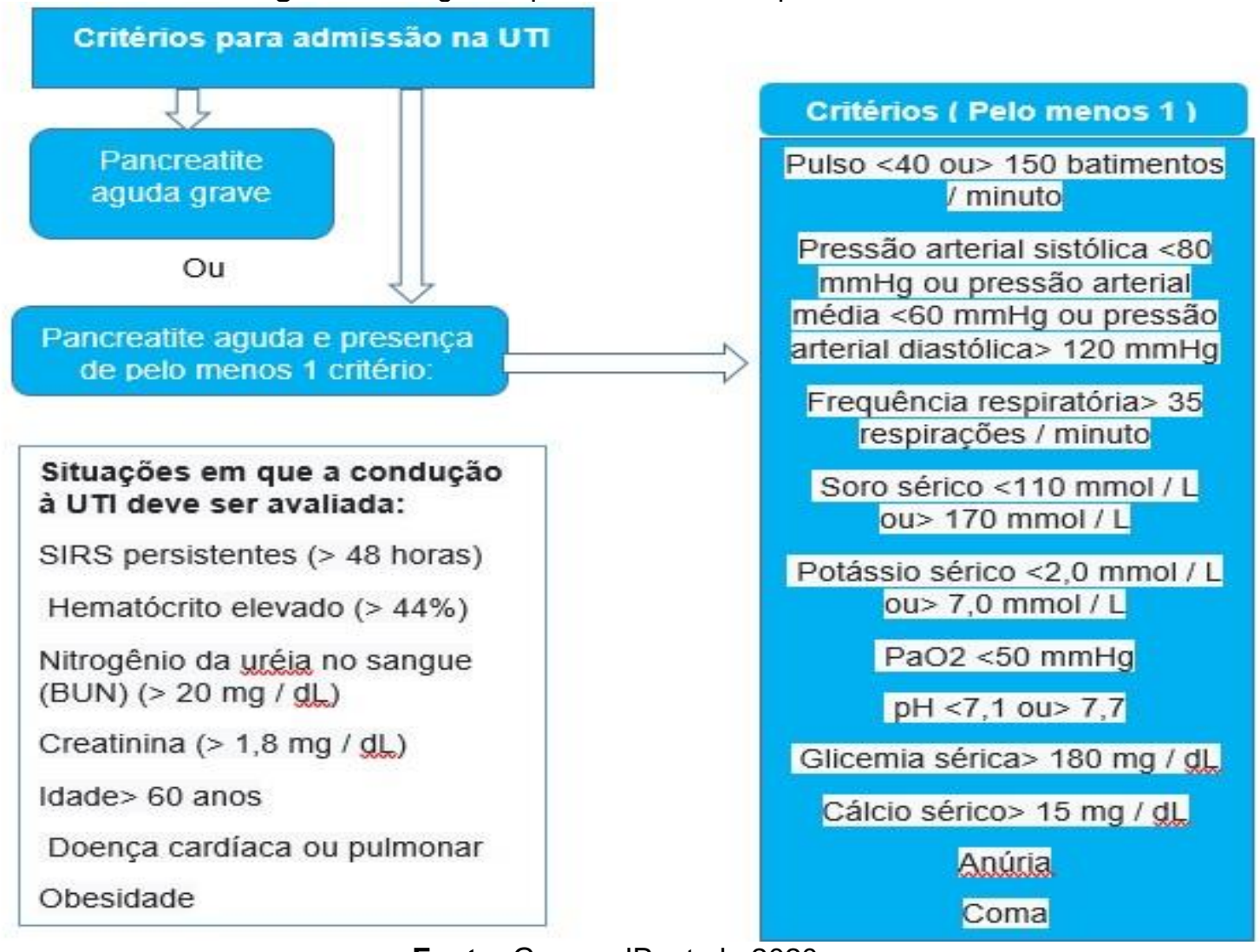

Fonte: Gomes JP, et al., 2020. 
Com relação à terapêutica, o paciente com PA precisa receber terapia de ressuscitação com fluidos. Associado a isso, medicamentos para controle da dor devem ser prescritos, além do suporte nutricional. Esses pacientes devem ser monitorados de perto nas primeiras 24 a 48 horas com avaliação dos seus dados vitais. A saturação de oxigênio deve ser mantida maior do que $95 \%$. A suplementação de oxigênio pode ser necessária. Caso seja menor do que $90 \%$, deve-se realizar a análise dos gases no sangue. Se a hipóxia persistir, é prudente o encaminhamento para a UTI (VEGE SS, 2020).

O débito urinário deve manter valores maiores do que 0,5 a $1 \mathrm{cc} / \mathrm{kg} / \mathrm{hora}$. É de grande importância a sua monitorização contínua (WORKING GROUP IAP/APA, 2013). Além disso, é imprescindível avaliar também os valores dos eletrólitos, especialmente, nas primeiras 48 a $72 \mathrm{~h}$ e realizar as correções necessárias (VEGE SS, 2020). A glicose deve ser monitorada e, tendo em vista o risco de infecção pancreática secundária e de alteração no controle nutricional associado à hiperglicemia, a glicemia deve ser corrigida caso esteja acima de $180 \mathrm{mg} / \mathrm{dL}$ (VEGE SS, 2020). Ademais, deve-se realizar medidas seriadas da pressão da bexiga para identificar possíveis pacientes acometidos pela Síndrome do Compartimento Abdominal (DE WAELE JJ, et al., 2011).

A reposição hídrica realizada nas primeiras 12 a 24 horas está relacionada a uma redução na morbimortalidade nos casos de PA (VEGE SS, 2020). A taxa dessa hidratação é ajustada com base na avaliação clínica, nos valores de hematócrito e nitrogênio da uréia no sangue (BUN) (WU BU, et al., 2011). Todos os pacientes com PA devem receber hidratação com solução cristaloide isotônica a uma taxa de 5 a $10 \mathrm{~mL} / \mathrm{kg} / \mathrm{hora}$. Exceto na presença de determinadas comorbidades cardíacas ou renais.

Nos casos em que haja hipotensão e taquicardia, recomenda-se a reposição de $20 \mathrm{~mL} / \mathrm{kg}$ de fluido intravenoso administrado por 30 minutos, seguido de $3 \mathrm{~mL} / \mathrm{kg} / \mathrm{hora}$ por 8 a 12 horas (GARDNER TB, et al., 2008; HAYDOCK MD, et al., 2013).

A reposição adequada de líquidos pode ser avaliada por uma melhora nos sinais vitais (frequência cardíaca < 120 batimentos/minuto, pressão arterial média entre 65 a $85 \mathrm{mmHg})$, débito urinário $(>0,5 \mathrm{a} 1 \mathrm{cc} / \mathrm{kg} / \mathrm{hora}) \mathrm{e}$ redução no hematócrito (meta de 35\% a 44\%) e uréia por mais de 24 horas (WORKING GROUP IAP/APA, 2013; WU BU, et al., 2010).

Antibióticos profiláticos para pacientes com PA não estão indicados. Esses medicamentos devem ser usados apenas na presença de infecção ou, caso haja suspeita, podem ser administrados até que os testes sejam realizados (CROCKETT SD, et al., 2018; TENNER S, et al., 2013). O uso de inibidores de protease (antirretrovirais) não são recomendados até o momento, pois não há dados concretos que comprovem redução considerável da mortalidade após o seu uso (TAKEDA K, et al., 1996).

Contrário ao que era postulado, em pacientes com PA leve ou moderada, a nutrição oral deve ser encorajada na medida em que haja tolerância para tal. Acreditava-se que o melhor manejo nutricional do doente era deixar o pâncreas em um estado de "repouso" e, assim, diminuir a inflamação vigente. Entretanto, a retomada alimentar precoce parece reduzir o tempo de permanência hospitalar. Todavia, alguns cuidados devem ser tomados, principalmente ao que tange o consumo de alimentos processados, uma vez que podem conter agentes que danificam a camada mucosa do trato gastrointestinal (MCCLAVE SA, 2019).

Os portadores da forma grave de PA e que necessitarão de internação em terapia intensiva, devem iniciar precocemente a nutrição enteral, dentro das primeiras 24-48 horas. Essa prática demonstrou diminuir o risco de infecções, bem como o tempo de permanência em hospital. Observou-se, também, que a nutrição enteral é preferível à parenteral, já que há menos inflamação da mucosa intestinal na primeira (MCCLAVE SA, 2019).

\section{Prognóstico}

A PA tem apresentação clínica e gravidade muito variável. Essa doença abrange desde formas leves com recuperação completa a formas graves, envolvendo necrose pancreática e peripancreática, abscessos, falência de múltiplos órgãos e casos fatais (RIBEIRO GFF, et al., 2017). 
O prognóstico da doença está relacionado com a forma com que ela evolui. A PA leve tem curso clínico bastante distinto da PA grave. A forma grave da doença está presente em até $30 \%$ dos casos. Seus índices de mortalidade variam em $1 \%$ na forma leve e até $20-30 \%$ na forma grave, correspondendo a 14a principal causa de morte de origem gastrointestinal (FUKUDA JK, et al., 2013).

Desta forma, correto diagnóstico, estabelecido de forma precoce, e a determinação de sua gravidade são de fundamental importância para o adequado manejo terapêutico desses doentes. Para isso, diversas classificações e critérios foram desenvolvidos no intuito de definir seu prognóstico (FUKUDA JK, et al., 2013).

Nesse sentido, destacam-se os critérios de Ranson, a dosagem da proteína C reativa, os critérios do APACHEII, os critérios tomográficos de Balthazar e a classificação de Atlanta. Os mais aplicados na prática clínica são os critérios de Ranson e APACHE-II (RIBEIRO GFF, et al., 2017).

A avaliação dos parâmetros clínicos e laboratoriais pode auxiliar muito em situações em que não há disponível outros métodos de avaliação mais precisos. Dados clínicos e laboratoriais de rotina e escores multifatoriais, mensurados na admissão e durante as primeiras 48h de internação, são utilizados para estimar a magnitude da resposta inflamatória à lesão, bem como para prever a necessidade ou não de suporte intensivo (FERREIRA AF, et al., 2014).

Nos últimos anos, o valor do hematócrito na admissão hospitalar tem sido relacionado com um pior prognóstico. Valores acima de $44 \%$ no momento da admissão configuram-se como de risco independente para necrose do órgão, enquanto que sua normalidade tem valor preditivo negativo para PAG maior que $95 \%$ (FERREIRA AF, et al., 2014). Quanto mais intensa for a resposta inflamatória, maior será a perda de fluidos para o terceiro espaço, causando hemoconcentração. No pâncreas, essa elevação do hematócrito causa débito da microcirculação que pode cursar com o desenvolvimento de necrose pancreática (NIEHUES GD, et al., 2017).

Tal como o hematócrito, valores de uréia sérica acima de $20 \mathrm{mg} / \mathrm{dl}$ foram associados a maior risco de óbito, bem como qualquer elevação de seus valores após o início do acompanhamento do paciente (FERREIRA AF, et al., 2015). Níveis de creatinina $>2 \mathrm{mg} / \mathrm{dl}$ após hidratação, plaquetas $<100.000 / \mathrm{mm} 3$, fibrinogênio $<100 \mathrm{mg} / \mathrm{dl}$, produtos de degradação do fibrinogênio $>80 \mathrm{mcg} / \mathrm{ml}$ e cálcio $<7,5 \mathrm{mg} / \mathrm{dl}$ também são indicadores de formas graves de tal patologia (FUKUDA JK, et al., 2013).

Dentre os grupos de critérios mais utilizados na prática clínica, destaca-se o de Ranson, que consiste em 11 parâmetros, 5 dos quais são avaliados na admissão e os restantes durante as primeiras $48 \mathrm{~h}$. A presença de 3 ou mais critérios nas $48 \mathrm{~h}$ da admissão, classifica a pancreatite como grave.

Há ainda outra interpretação relacionando a quantidade de critérios presentes com a probabilidade de mortalidade, sendo que uma pontuação obtida entre 0 e 2 representa mortalidade de $2 \%$. Porém, pontuação entre 3 a 4 pontos aumenta a chance de óbito para 15\%. E, não obstante, pontuação entre 5 a 6 alcança índice de $40 \%$ de mortalidade podendo chegar até $100 \%$ quando obtido escore com 7 a 8 pontos (FERREIRA AF, et al., 2014).

Outro critério de suma importância é o de APACHE-II, um dos mais utilizados para estratificação de gravidade e risco de mortalidade da PA. Esse critério conta com 12 parâmetros de avaliação, além de pontuação extra baseada na idade e na presença de doenças crônicas. Dentre as suas vantagens, destaca-se o fato de poder ser calculado dentro das primeiras 24 horas da admissão do paciente no hospital e de poder ser realizado diariamente na avaliação da evolução do paciente. A adição de IMC para a pontuação APACHE-II - conhecido como Apache$\mathrm{O}$ - adiciona um ponto para IMC de $>25$ a $30 \mathrm{Kg} / \mathrm{m} 2$ e dois pontos para IMC $>30 \mathrm{Kg} / \mathrm{m} 2$ (FERREIRA AF, et al., 2014).

\section{Complicações}

As complicações da pancreatite aguda são variadas e podem ser separadas entre sistêmicas e locais, objetivando uma abordagem mais eficiente desses quadros que podem ser graves. 


\section{Complicações sistêmicas}

Quadro 1 - Complicações sistêmicas da pancreatite aguda.

\begin{tabular}{|c|c|}
\hline Hipovolemia & $\begin{array}{l}\text { Frequente na pancreatite aguda; hidratação parenteral e/ou expansores como } \\
\text { albumina melhoram o prognóstico. }\end{array}$ \\
\hline SNC & $\begin{array}{l}\text { Sonolência e confusão mental podem estar presentes. Encefalopatia pancreática é } \\
\text { uma complicação rara gerada pelo papel desmielinizante da lipase circulante. }\end{array}$ \\
\hline Aparelho Respiratório & $\begin{array}{l}\text { Pode-se ter um comprometimento pulmonar com consequente hipoxemia e } \\
\text { insuficiência respiratória. }\end{array}$ \\
\hline Aparelho Cardiovascular & $\begin{array}{l}\text { A liberação de cininas gera uma resposta inflamatória sistêmica, causando } \\
\text { vasodilatação. Esta, associada à hipovolemia, são responsáveis pelo quadro de } \\
\text { taquicardia e hipotensão arterial. }\end{array}$ \\
\hline Manifestações Renais & $\begin{array}{l}\text { Os variados processos de agravo sistêmico podem levar a uma má perfusão renal, } \\
\text { podendo ocasionar falência pré-renal }\end{array}$ \\
\hline Hemorragia Gastrointestina & $\begin{array}{l}\text { Frequente devido a injúria aguda à mucosa gástrica. Pode ser decorrente também de } \\
\text { condições como pseudoaneurismas das artérias esplênica ou gastroduodenal, } \\
\text { perfurações de abscessos em tubo digestivo e colite isquêmica. }\end{array}$ \\
\hline $\begin{array}{l}\text { Alterações metabólicas e } \\
\text { eletrolíticas }\end{array}$ & $\begin{array}{l}\text { Descompensação mediante destruição aguda da glândula pancreática, com ação } \\
\text { hiperglicemiante. Algumas condições podem determinar uma baixa no Magnésio } \\
\text { presente no soro. }\end{array}$ \\
\hline Disfunção hepatobiliar & $\begin{array}{l}\text { Hiperbilirrubinemia conjugada e elevação de enzimas hepáticas devido icterícia } \\
\text { obstrutiva e/ou lesão hepática. }\end{array}$ \\
\hline Coagulopatias & $\begin{array}{l}\text { Coagulação intravascular disseminada que é relacionada com isquemia pancreática. } \\
\text { Com frequência, a avaliação hematológica demonstra plaquetopenia e níveis } \\
\text { elevados de produtos de degradação da fibrina. }\end{array}$ \\
\hline Retinopatia & $\begin{array}{l}\text { A Retinopatia de Purtscher expressa diminuição súbita ou perda total da visão. } \\
\text { Regride espontaneamente. }\end{array}$ \\
\hline Nódulos subcutâneos & $\begin{array}{l}\text { Lesão rara na pancreatite aguda e que tende a ser mais frequente em região de } \\
\text { membros inferiores }\end{array}$ \\
\hline $\begin{array}{l}\text { Manifestações } \\
\text { osteoarticulares }\end{array}$ & $\begin{array}{l}\text { Também raras, podem se traduzir em poliartrite, necrose asséptica de ossos, lesões } \\
\text { osteolíticas e reações de periósteo. }\end{array}$ \\
\hline
\end{tabular}

Fonte: Gomes JP, et al., 2020. Baseado em: Dani R e Passos MCF, 2011; Silva RA, et al., 2020.

\section{Complicações locais}

Quadro 2 - Complicações locais da pancreatite aguda.

\begin{tabular}{|c|l|}
\hline Infecção e septicemia & $\begin{array}{l}\text { Infecção local "pode manifestar-se de três maneiras: como necrose infectada, como } \\
\text { abscesso pancreático ou como pseudocisto infectado, que também é um abscesso.” } \\
\text { Em todas as formas existe a possibilidade da ocorrência de septicemia. }\end{array}$ \\
\hline Abscesso pancreático & $\begin{array}{l}\text { “Coleção de material purulento encapsulado por uma parede fibrosa localizado no } \\
\text { pâncreas ou na região retropancreática.” Aparece "geralmente após quatro a seis } \\
\text { semanas do início do quadro e não contém necrose pancreática." O tratamento baseia- } \\
\text { se na drenagem cirúrgica, desbridamento de tecidos e limpeza local. }\end{array}$ \\
\hline $\begin{array}{c}\text { Pseudocisto pancreático } \\
\text { o que o diferencia de um cisto pancreático verdadeiro." Ocorre de 2\% a 8\% dos casos. }\end{array}$ \\
\hline $\begin{array}{c}\text { Ascite e ruptura de ducto } \\
\text { pancreático }\end{array}$ & $\begin{array}{l}\text { Geralmente ocorre em quadros de pancreatite aguda com uma quantidade necrótica } \\
\text { significativa de parênquima pancreático. }\end{array}$ \\
\hline $\begin{array}{c}\text { Obstrução do tubo } \\
\text { digestivo }\end{array}$ & $\begin{array}{l}\text { Pode ocorrer por paresia reflexa, extensão de processo inflamatório ou compressão } \\
\text { cística. }\end{array}$ \\
\hline Obstrução biliar & Causado por edema da cabeça do pâncreas ou pseudocistos \\
\hline Necrose do cólon & $\begin{array}{l}\text { Com a evolução necrótica pode haver peritonite e perfuração; é preconizado tratamento } \\
\text { cirúrgico }\end{array}$ \\
\hline $\begin{array}{c}\text { Ruptura de } \\
\text { pseudoaneurisma }\end{array}$ & $\begin{array}{l}\text { Causado pelas injúrias provocadas pelas enzimas na parede arterial pancreática. } \\
\text { Tratamento é cirúrgico; por vezem embolização }\end{array}$ \\
\hline Ruptura esplênica & “Complicação raríssima, parece associar-se à trombose aguda da veia esplênica”. \\
\hline
\end{tabular}

Fonte: Gomes JP, et al., 2020. Baseado em: Dani R e Passos MCF, 2011; Silva RA, et al., 2020. 


\section{CONSIDERAÇÕES FINAIS}

Tendo em vista a expressiva incidência de PA alcoólica (30\% das PAs), seus efeitos ao indivíduo e ao sistema de saúde, observou-se a importância de realizar uma atualização dos pontos principais relativos a esse tema. Nesse sentido, a análise dos achados clínico-laboratoriais é essencial para se determinar a gravidade do quadro e propor o melhor tratamento, de forma a garantir que os pacientes críticos sejam admitidos em unidades de terapia intensiva, nas quais o manejo desses indivíduos baseia-se em prover ressuscitação agressiva com fluidos, controle álgico e suporte nutricional adequado. O prognóstico da doença é variável de acordo com a sua gravidade e possível ocorrência de algumas complicações, como disfunção hepatobiliar, coagulopatias e septicemia. Por fim, destaca-se que os critérios de Ranson e APACHE-II são de grande utilidade na prática clínica para análise do prognóstico do quadro analisado.

\section{REFERÊNCIAS}

1. AGUILAR A, et al. Pancreatitis aguda: fisiopatología y manejo inicial. Acta Med Costarric. 2019; 61(1): 13-21.

2. ÁLVARES LGGS, et al. Perfil Clínico-Epidemiológico de pacientes com pancreatite aguda em um hospital público de São Luís, Maranhão. Revista de Pesquisa em Saude 2014; 14(2): 109-112.

3. BARRY K. Chronic Pancreatitis: Diagnosis and Treatment. Am Fam Physician, 2018; 97(6): 385-393.

4. BOLLEN TL, et al. Atualização sobre pancreatite aguda: ultra-som, tomografia computadorizada e ressonância magnética. Ultrasom Semin CT MR, 2007; 28(1): 371-83.

5. BRASILEIRO FILHO G. Bogliolo patologia. 9. ed. Rio de Janeiro: Guanabara Koogan, 2016; 1542p.

6. BURROWES DP, et al. Utilidade do ultrassom na pancreatite aguda. Abdom Radiol, 2020; 45(5), 1253-1264.

7. CHATILA AT, et al. Avaliação e tratamento da pancreatite aguda. Casos do mundo J Clin, 2019; 7(9): 1006-1020.

8. CHOWDHURY P, GUPTA P. Pathophysiology of alcoholic pancreatitis: an overview. World J Gastroenterol, 2006;12(46): 7421 7427.

9. CROCKETT SD, et al. American Gastroenterological Association Institute Guideline on Initial Management of Acute Pancreatitis. Gastroenterology, 2018; 154(4): 1096-1101.

10. DANI R, PASSOS MCF. Gastroenterologia Essencial. 4. ed. Rio de Janeiro: Guanabara Koogan, 2011. 1324p.

11. DE WAELE JJ, et al. Intra-abdominal Hypertension and Abdominal Compartment Syndrome. Am J Kidney Dis, 2011; 57(1): 159169

12. DOHERTY GM. Current Cirurgia: Diagnóstico e Tratamento. 14. ed. São Paulo: AMGH editora Ltda, 2017. 1408p.

13. FERREIRA AF, et al. Fatores preditivos de gravidade da pancreatite aguda: quais e quando utilizar?. ABCD, arq. bras. cir. dig. 2015; 28(3): 207-211. 14. FUKÜDA JK, et al. Prognóstico dos casos de pancreatite aguda pelo escore de PANC 3. ABCD, arq. bras. cir. dig., 2013; 26(2):
133-135.

15. GARDNER TB, et al. Fluid resuscitation in acute pancreatitis. Clin Gastroenterol Hepatol 2008; 6(10): $1070-1076$.

16. HAYDOCK MD, et al. Fluid therapy in acute pancreatitis: anybody's guess. Ann Surg 2013; 257(2): $182-188$.

17. KLOCHKOV A, et al. Alcoholic Pancreatitis. StatPearls [Internet], Updated Jan 232020.

18. MCCLAVE SA. Factors that worsen disease severity in acute pancreatitis: implications for more innovative nutrition therapy. Nutr Clin Pract, 2019; 34(1): 43-48.

19. MCPHERSON RA, PINCUS, MR. Diagnósticos Clínicos e Tratamento por Métodos Laboratoriais de Henry. 21. ed. Barueri, SP: Editora Manole, 2012. 1664p.

20. NIEHUES GD, et al. Avaliação clínico-laboratorial e o prognóstico da pancreatite aguda biliar. Arquivos Catarinenses de Medicina, 2017; 46(2): 02-14.

21. PHAM A, FORSMARK C. Chronic pancreatitis: review and update of etiology, risk factors, and management. F1000 Research, 2018. 7(Faculty Rev): 607-618.

22. RAHMAN A, et al. Clinical classification and severity scoring systems in chronic pancreatitis: a systematic review. Dig Surg, 2020; 37(3):181-191.

23. RIBEIRO GFF, et al. Etiologia e mortalidade por pancreatite aguda: uma revisão sistemática. Arquivos Catarinenses de Medicina, 2017; 46(4): 168-181.

24. SANTOS JS, et al. Pancreatite aguda: atualização de conceitos e condutas. Medicina (Ribeirão Preto), $2003 ; 36(3): 266-282$.

25. SILVA RA, et al. Quando e como tratar as complicações na necrose pancreática infectada. ABCD, arq. bras. cir. dig., 2010; 23(4): $270-274$.

26. SILVERTHORN, DU. Fisiologia humana: uma abordagem integrada. 7. ed. Porto Alegre: Artmed, 2017. 960p

27. SINGH VK, et al. An assessment of the severity of interstitial pancreatitis. Clin Gastroenterol Hepatol, 2011; 9(12): 1098-1103.

28. SINGH P, GARG P. Pathophysiological mechanisms in acute pancreatitis: current understanding. Indian $\mathrm{J}$ Gastroenterol. 2016;35(3):153-66.

29. TAKEDA K, et al. Continuous regional arterial infusion of protease inhibitor and antibiotics in acute necrotizing pancreatitis. Am $\mathrm{J}$ Surg, 1996; 171(4): 394-398.

30. TENNER S, et al. American College of Gastroenterology guideline: management of acute pancreatitis. Am J Gastroenterol, 2013; 108(9): 1400-1415.

31. VAN SANTVOORT HC, et al. A conservative and minimally invasive approach to necrotizing pancreatitis improves outcome. Gastroenterology, 2011; 141(3): 1254-1263.

32. VEGE SS. Management of acute pancreatitis. Post TW, ed. UpToDate, 2020.

33. WORKING GROUP IAP/APA. Acute Pancreatitis Guidelines. IAP/APA evidence-based guidelines for the management of acute pancreatitis. Pancreatology, 2013; 13(4-supp2): e1-e15

34. WU BU, et al. Acute pancreatitis part l: approach to early management. Clin Gastroenterol Hepatol, 2010; 8(5): 410-416.

35. WU BU, et al. Lactated Ringer's solution reduces systemic inflammation compared with saline in patients with acute pancreatitis. Clin Gastroenterol Hepatol, 2011; 9(8): 710-717.

36. YADAV D, Lowenfels AB. The epidemiology of pancreatitis and pancreatic cancer. Gastroenterology 2013; 144(6): 1252-1261

37. ZATERKA S, EISIG JN. Tratado de gastroenterologia: da graduação à pós-graduação. São Paulo: Atheneu, FBG, 2011.1260 p.

38. ZHAO G, et al. Effects of different resuscitation fluid on severe acute pancreatitis. World J Gastroenterol, 2013; 19(13): 2044-2052. 